\title{
G

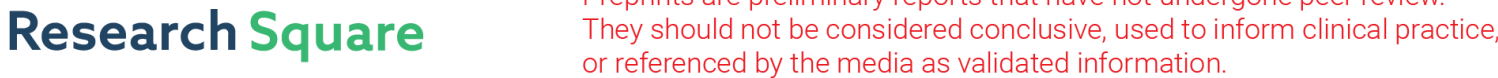 \\ Coronavirus in the Skin of the Pangolin: Implications for SARS-CoV-2 Transmission?
}

\section{Siwei Deng}

Wenzhou-Kean University: Kean University - Wenzhou Campus

Xuechen Tian

Wenzhou-Kean University: Kean University - Wenzhou Campus

Robert Belshaw

Wenzhou-Kean University: Kean University - Wenzhou Campus

Jinfeng Zhou

China Biodiversity Conservation and Green Development Foundation (CBCGDF)

\section{Siyuan Zhang}

China Biodiversity Conservation and Green Development Foundation (CBCGDF)

\section{Yixin Yang}

Wenzhou-Kean University: Kean University - Wenzhou Campus

\section{Chang Huang}

Wenzhou-Kean University: Kean University - Wenzhou Campus

\section{Weikang Chen}

Wenzhou-Kean University: Kean University - Wenzhou Campus

Hailu Qiu

Wenzhou-Kean University: Kean University - Wenzhou Campus

Siew Woh Choo (D cwoh@wku.edu.cn)

Wenzhou-Kean University: Kean University - Wenzhou Campus

\section{Research Article}

Keywords: COVID-19, coronavirus, SARS-CoV-2, pangolins, endogenous viral genes, percutaneous transmission

Posted Date: March 23rd, 2021

DOI: https://doi.org/10.21203/rs.3.rs-354445/v1

License: (c) (i) This work is licensed under a Creative Commons Attribution 4.0 International License. Read Full License 


\section{Abstract}

COVID-19 has become a global pandemic caused by SARS-CoV-2 infection. Dermatologic manifestations are not uncommon in COVID-19 patients and the virus has been reported within some. However, the role of the virus and the host response in these cutaneous manifestations remain poorly studied. The pangolin coronavirus ( $\mathrm{pCoV}$ ) is a model for studying SARS-CoV-2 because of its genetic relatedness and similarity of disease characteristics. Here we report the presence of $\mathrm{pCoV}$ in pangolin skin manifestations and present an in-depth analysis of the transcriptional response to the infection. COVID-19-related pathways were up-regulated, but interferon epsilon (IFNE)-related pathways were not differentially regulated (consistent with the pseudogenisation of IFNE and IFIH1 in the pangolin). Endogenous retroviral genes tended to be down-regulated, indicating their importance in CoV infection. We believe our study provides further support for a potentially overlooked percutaneous transmission route of SARSCoV-2 infection.

\section{Introduction}

Coronavirus disease 2019 (COVID-19), caused by severe acute respiratory syndrome coronavirus 2 (SARS-CoV-2) infection, has become a global pandemic ${ }^{1}$. At the beginning of the pandemic in early 2020, it was proposed that the Malayan pangolin (Manis javanica) was an intermediate host of bat-to-human transmission of SARS-CoV-2 ${ }^{2,3}$, but subsequent sequence analysis has shown this not to be the case ${ }^{4}$. Nevertheless, the pangolin coronavirus ( $\mathrm{pCoV}$ ) is closely related to SARS-CoV-2 and may provide insights into its pathology.

Clinical and histopathological studies of COVID-19 patients reported some dermatologic manifestations such as petechiae (a rash and haemorrhagic dot-like areas) ${ }^{5-8}$. The reason for the skin eruption in COVID19 patients is unknown, but may be due to a SARS-CoV-2 interaction with skin ${ }^{5-8}$. Also, it has been suggested that angiotensin-converting enzyme 2 (ACE2), which is used by SARS-CoV-2 to enter the host ${ }^{9}$, can be highly expressed in keratinocytes ${ }^{10,11}$. These results suggested that percutaneous transmission might be an overlooked route of SARS-CoV-2 infection.

In this study, we investigated a possible pCoV skin infection in a Malayan pangolin named "Dahu" seized by customs in the Guangdong province (China) before dying ${ }^{12}$. We previously reported that Dahu's brain (cerebrum and cerebellum) and lungs were infected by $\mathrm{pCoV}^{12}$ and here we confirm that Dahu's skin was also infected. With RNA-seq data, we found that the most up-regulated molecular pathway was the same SARS-CoV-2-associated pathway previously identified in the lungs of infected humans ${ }^{13}$. The main differences in pathway dysregulation could be attributed to the pangolin's unique immune characteristics, namely inactivation of the interferon epsilon (IFNE) and interferon-induced with helicase $\mathrm{C}$ domain 1 $\left(\mathrm{IFIH} 1\right.$, also known as MDA5) ${ }^{14,15}$. We also found that many endogenous retroviral (ERV) genes were differentially expressed in Dahu skin, possibly because of the pCoV infection, suggesting that they might 
play a role in $\mathrm{CoV}$ infection. These findings indicate that $\mathrm{pCoV}$ was replicating within the skin tissue of Dahu.

\section{Methods}

\section{Overview}

We present an overview of the Methods, with further methodological details provided in the Supplementary Information. Briefly, Dahu skin tissue was harvested by veterinary officers at Guangzhou Leader Animal Hospital. RNA was extracted from a lesion in the skin of the neck using Qiagen RNeasy Mini Kit and sequenced using Illumina HiSeq with a $2 \times 150 \mathrm{bp}$ paired-end strategy. We compared our infected skin with published data from the healthy skin of another pangolin (project accession PRJNA283328; run SRR3923846). The read counts, normalised expression values and differentially expressed genes (DEGs) were generated according to the Cufflinks pipeline ${ }^{16}$. For comparative analysis, the list of DEGs was compared with the gene list generated from human COVID-19 patients from the study of Blanco-Melo et al. (project accession PRJNA615032) ${ }^{17}$. Functional enrichment analyses including gene set enrichment analysis (GSEA) and over-representation analysis (ORA) were performed using clusterProfiler v3.18.0 ${ }^{18}$. ERV elements were identified using TBLASTN ${ }^{19}$ and in-house scripts. Phylogenetic trees were constructed using MEGA- $X^{20}$. Sequences were aligned using the MUSCLE algorithm ${ }^{21}$. The maximum-likelihood trees were inferred using the Tamura-Nei DNA substitution model and nodal support was estimated using 1,000 replicates.

\section{Results}

\section{The presence of pCoV subgenomic mRNA in the skin}

To test whether Dahu skin was infected by pCoV (in addition to its lungs and brains ${ }^{12}$ ), we searched for viral sequences among the transcriptome data. A total of 193 reads mapped to the reference $\mathrm{pCoV}$ genome (Figure S1). The distribution of these mapped reads was consistent with the corresponding locations of the subgenomic mRNAs ${ }^{22}$. We also observed an individual read that spanned precisely the splicing sites: this read was $150 \mathrm{bp}$ in length and its $5^{\prime} 47 \mathrm{bp}$ mapped to the $5^{\prime}$ region of the pCoV genome, while its 3' 103 bp mapped to the 3' region of the same genome. This read indicates that the CoV RNA has been processed in the host cell ${ }^{23}$. Consistently, we detected the $\mathrm{N}$ gene (which is used in diagnostic human SARS-CoV-2 testing) in all the tested Dahu organ using qRT-PCR ${ }^{12}$, including the skin. To confirm the identity of the viral RNA in Dahu skin, we compared consensus sequences from our mapped reads with CoV from other species (Figure 1; Table S1). We observed that the pCoV genome and genes from Dahu skin were almost identical (sequence identity $=99.2-100 \%$ ) to the counterparts from another pangolin, Guangdong pCoV isolate MP78924, confirming the presence of pCoV RNA in the Dahu skin. 


\section{Dahu skin transcriptional responses to pCoV}

To explore the host skin transcriptional responses, we identified 3,201 DEGs (1,810 up-regulated and 1,391 down-regulated) in Dahu skin (Table S2). Consistent with the results observed in human COVID-19 patients ${ }^{13}$, we found terms associated with immunity processes were significantly enriched and upregulated, such as terms related to non-interferon cytokine production, T-cell, neutrophil, myeloid cell, and mast cell differentiation and activation (Figure 2D). Apoptotic signalling pathways were negatively regulated, especially apoptotic processes of immune cells, such as myeloid cells and leukocytes. Also, rRNA metabolic process, RNA splicing, mRNA transcription, translational processes, and protein folding were also up-regulated. Specifically, we found genes associated with viral transcription and viral gene expression were up-regulated. Other terms, including complements and cellular response to biotic stimulus were up-regulated, while cell cycle processes were down-regulated.

Strikingly, we found that the coronavirus disease-COVID-19 pathway was the most significant upregulated pathway (Figure 2E; Table S3; Figure S2). Ribosomal proteins were up-regulated (Figure S3A; Figure 2E; Table S3) as CoV needs ribosome frameshifting to translate and replicate ${ }^{25,26}$. It has been shown that the nonstructural protein 1 of the SARS-CoV-2 is a major virulence factor that interferes with host RNA translation by binding to 40 S ribosomal subunit ${ }^{27,28}$. Li summarised the functional relationships between host ribosomal proteins and viral infection ${ }^{29}$, and suggested most interactions are beneficial for viral protein translation and replication. We found ribosomal proteins that are crucial for viral infection were up-regulated in Dahu skin (Figure S3), such as RPL3 ${ }^{30}$, RPL18 $8^{31-34}$, and RPL24 ${ }^{35}$. Moreover, we found RPL9 and RPL22 which help virus particle assembly and viral gene expression, were up-regulated in Dahu-skin, but not human patient lungs (Figure S3B), probably a new strategy to promote its replication in pangolin ${ }^{36,37}$.

Other up-regulated pathways include pathways in cancer, TNF signalling pathway, complement and coagulation cascades, chemokine signalling pathway, immunity pathway, and viral interaction/infection related pathways (Figure 2E; Table S3). Furthermore, the DEGs were down-regulated in pathways associated with linoleic acid and lipid metabolism (Figure 2E; Table S3). Again, these host responses are consistent with the core pathways identified in the human SARS-CoV-2 infection ${ }^{13}$.

\section{Comparative analysis of transcription in Dahu skin and human lungs}


We further investigated the similarities and differences of host responses between Dahu skin and the human lungs by comparing DEG lists between our study and an external human lung study ${ }^{17}$. Our comparative analysis revealed 366 DEGs shared between species (i.e., 'common DEGs' below), 2,835 Dahu skin-specific DEGs, and 1,527 human lung-specific DEGs. As anticipated, the common DEGs were enriched in coronavirus diseases-COVID-19 pathway, followed by MAPK signalling pathway, apoptosis, Ctype lectin receptor signalling pathway and Kaposi sarcoma-associated herpesvirus infection. These findings are consistent with the pCoV infection in Dahu skin (Figure 3A).

Interestingly, interferon-specific responses were exclusively up-regulated in human lungs compared to Dahu skin. We found interferon responses (e.g., interferon-gamma production) significantly enriched and up-regulated in human lung-specific DEGs (Figure 3C-D; Table S4), but not in Dahu skin-specific DEGs (Table S5). We also downloaded genes related to interferon signalling pathways in Reactome pathway database ${ }^{38,39}$ and found 131 genes were expressed in Dahu skin and/or human lungs (Figure 3E; Table S6). Among them, 102 genes were expressed in human lungs, while 88 genes were expressed in Dahu skin (Figure 3E). We found 42 of them (37 up-regulated and 5 down-regulated) were differentially expressed in human lungs, but none of them was differentially expressed in Dahu skin. It has been reported that pangolins have unique immune characteristics ${ }^{14,15}$ : both IFNE and IFIH1 (a cytoplasmic RNA sensor that helps initiates the innate immune response to viral infection) genes have been pseudogenised. IFNE plays an important antiviral role in epithelial cells ${ }^{40-46}$. Therefore, IFNE-deficient pangolin skin may be susceptible to CoV infection. Therefore, it indicates pangolin might adopt alternative strategies to fight against CoV infection over evolutionary time.

In our comparative analysis, we found three enriched pathways in Dahu skin-specific DEGs (Figure 3B), in which malaria and Staphylococcus aureus infection pathways were up-regulated in Dahu skin-specific DEGs, while arachidonic acid (AA) metabolism pathways were down-regulated. These pathways may indicate unique pangolin host skin responses. Malaria pathway is commonly up-regulated after SARSCoV-2 infection ${ }^{13}$, and anti-malarial drugs have shown effects on inhibiting SARS-CoV-2 replication ${ }^{47}$. In addition, we discovered that malaria pathway was also up-regulated in human lungs. As it is one of the three Dahu skin-specific pathways, it indicates malaria pathway was further up-regulated in Dahu skin. Consistent with other up-regulated inflammatory responses, we observed Staphylococcus aureus infection pathway was specifically up-regulated in Dahu skin.

In addition, the AA metabolism pathways were down-regulated in Dahu skin-specific DEGs (Figure 3B), indicating that these pathways were suppressed by pCoV infection. It is known that AA pathways have inhibitory effects on coronavirus replication, suggesting that lipid metabolism could be a druggable target of coronavirus-infected patients ${ }^{48}$. Therefore, $\mathrm{pCoV}$ might also suppress AA metabolism pathway in pangolin skin to benefits its replication. 


\section{Responses of endogenous retrovirus (ERV) gene expression in the pCoV skin infection}

Then, we investigated the relationships between host ERV gene expression and pCoV infection. We identified 6,076 ERV genes by screening 3,162 known viral proteins from Swiss-Prot across the pangolin genome (Table S7). We found 466 genes expressed in infected and/or non-infected pangolin skin, in which 348 of them (81 up-regulated versus 267 down-regulated) were differentially expressed (Figure 4A; Figure S4; Table S8), suggesting that the exogenous pCoV might suppress the expression of ERV genes after infection to benefit its replication. We found most of the ERV DEGs were env (43\%), pol (31\%), and gag (16\%) (Figure 4B; Table S8), while the compositions in the genome were $28 \%, 38 \%$, and $20 \%$, respectively (Figure S5A; Table S8). The most abundant group of ERV DEGs were most closely related to mouse intracisternal A-particle (IAP) viruses (Figure 4D; Table S8). Mouse and Hamster IAP viruses make up $19 \%$ of the ERV DEGs, while only $11 \%$ of the IAP sequences are found in the pangolin genome (Figure S5C; Table S7), which may suggest the importance of IAPs in CoV infection and their possible interaction with the virus.

\section{Discussion}

In this study, pCoV RNA was found in the Dahu skin. This Cov was almost identical to pCoV MP789 from the Guangdong pangolin. Dahu and the Guangdong pangolin MP789 were seized by Guangdong antismuggling bureaus after being smuggled into China ${ }^{12}$ and kept together at Guangdong wildlife rescue centre. Therefore, it is possible that both pCoVs originated from the same source ${ }^{12}$.

Our functional enrichment analyses are generally consistent with the results observed in human patients with SARS-CoV-2 infection ${ }^{13}$. After being infected, the immune responses were activated via immune cells (lymphocytes, leukocytes, myeloid cells, neutrophils, and mast cells), stimulating non-interferon cytokine production and preventing immune cell apoptosis ${ }^{49,50}$. It has been shown that the cytokine storm is also an early feature after SARS-CoV-2 infection ${ }^{51}$, which can be cross-validated by up-regulating rRNA metabolic process, RNA splicing, mRNA transcription, translational processes, and protein folding. Also, the virus exploits host cell machinery to proliferate in the host ${ }^{52}$, which up-regulates genes associated with viral transcription and viral gene expression. Complement activation and hypercoagulation are frequently accompanied by SARS-CoV-2 infection ${ }^{53}$. Here, we also observed the activation of complement pathways in Dahu skin. Furthermore, cell cycle processes were suppressed in pangolin skin. It has been shown that $\mathrm{CoV}$ can arrest cell cycle to boost viral replication efficiency ${ }^{54}$ through mechanisms such as regulating through cyclin-CDK complex ${ }^{55,56}$, p53-dependent pathway ${ }^{57,58}, \mathrm{~N}$ protein of coronavirus ${ }^{59-61}$, and directly interacting host cell cycle proteins ${ }^{62,63}$.

At the pathway level, consistent with SARS-CoV-2 infection, our analysis showed that COVID-19 pathway, immunity and inflammation (except for IFN-related pathways), cell proliferation, and coagulation pathways were the most significant up-regulated pathways in the Dahu $\operatorname{skin}^{13}$. In the CoV-infected 
pangolin skin, the interferon-specific pathways were not enriched, and the expressions of many interferon pathway-related genes were undetectable or/and not significantly differentially expressed. In other placental mammals, IFNE provides the first line of defence in the skin and mucosa-protected organs (e.g., lungs) and senses viral infection by triggering downstream genes involved in the interferon pathways ${ }^{40-}$ 46. Therefore, the IFNE-mediated pathways, including interferon-stimulated gene responses, are unlikely to be activated or up-regulated in the naturally IFNE- and IFIH1-deficient pangolins upon CoV infection ${ }^{14}$.

In healthy pangolin skin, many ERV genes were expressed, indicating their biological significance. We believe that these ERV genes are beneficial to the host, such as to boost host's immunity ${ }^{64-67}$, which is especially important in IFNE-deficient pangolins. Our data showed that most of the ERV genes were down-regulated after being infected, leading to our speculation that pCoV might directly or indirectly suppress the ERV genes to benefit its proliferation.

pCoV might infect the skin cells via the known receptor ACE2. However, the expression of ACE2 in Dahu skin was extremely low or undetectable. It is consistent with low interferon level because ACE2 is stimulated by interferon ${ }^{68}$. Also, low RNA level does not mean ACE2 is not present. We used bulk RNA-seq in this study, but ACE2 is only expressed in the skin keratinocytes, and might only be detectable using single-cell technology ${ }^{10,11}$. In addition, the ACE2 RNA expression is low even in the healthy human lungs, where the protein is thought to be the main receptor ${ }^{69}$. Another possibility is that $\mathrm{pCoV}$ might infect the skin cells through alternative receptors such as DPP4, which is expressed in infected Dahu skin. Studies have shown that DPP4 is a potential binding target of SARS-CoV-2, leading to suitable therapeutic possibilities $^{70,71}$. Gianotti, Zerbi, and Dodiuk-Gad ${ }^{5}$ have demonstrated clinical features of SARS-CoV-2 infected patient skin, and they hypothesised that the patient skin eruption might be due to the viral interaction with the skin. We cannot rule out the possibility that the skin of these patients was indeed infected by SARS-CoV-2 and novel mechanisms may exist to assist CoV in infecting the skin.

A limitation of this study is that our observations are only based on one CoV-infected pangolin. We are also unsure whether our observations are caused by the unique pseudogenised IFNE and IFIH1, although this might resemble the response of people who have weakened immunity. A possible cause of our observations is contamination by pCoV-infected blood. However, a recent study showed the enrichment of representative pathways such as porphyrin and chlorophyll metabolism, oxidative phosphorylation, and tryptophan metabolism in human SARS-CoV-2 infected blood transcriptome ${ }^{72}$, but we did not observe these pathways enriched in the Dahu skin transcriptome. We therefore suggest that the presence of $\mathrm{pCoV}$ and the systematic host's responses to CoV infection observed in Dahu skin is unlikely to be due to blood contamination.

\section{Conclusion}

We report the presence of pCoV subgenomic (spliced) mRNAs, which only occur in infected cells, and transcriptomic hallmarks of host response to CoV infection in Dahu skin. We suggest it is the strongest 
evidence so far for CoV infection in the skin of a placental mammal. Also, the pathway dysregulation was consistent with CoV infection of an organism lacking IFNE and IFIH1, and we observed the downregulation of ERV genes. This study emphasises the possibility of SARS-CoV-2 transmission via skin in humans.

\section{References}

1. Zhou, P. et al. A pneumonia outbreak associated with a new coronavirus of probable bat origin. Nature 579, 270-273 (2020).

2. Michel, C. J., Mayer, C., Poch, O. \& Thompson, J. D. Characterization of accessory genes in coronavirus genomes. Virol. J. 17, 131 (2020).

3. Lopes, L. R., de Mattos Cardillo, G. \& Paiva, P. B. Molecular evolution and phylogenetic analysis of SARS-CoV-2 and hosts ACE2 protein suggest Malayan pangolin as intermediary host. Braz. J. Microbiol. Publ. Braz. Soc. Microbiol. 51, 1593-1599 (2020).

4. Zhao, J., Cui, W. \& Tian, B.-P. The Potential Intermediate Hosts for SARS-CoV-2. Front. Microbiol. 11, 580137 (2020).

5. Gianotti, R., Zerbi, P. \& Dodiuk-Gad, R. P. Clinical and histopathological study of skin dermatoses in patients affected by COVID-19 infection in the Northern part of Italy. J. Dermatol. Sci. 98, 141-143 (2020).

6. Gianotti, R., Barberis, M., Fellegara, G., Galván-Casas, C. \& Gianotti, E. COVID-19 related dermatosis in November 2019. Could this case be Italy's patient zero? Br. J. Dermatol. (2021) doi:10/ghtn5s.

7. Seirafianpour, F. et al. Cutaneous manifestations and considerations in COVID-19 pandemic: A systematic review. Dermatol. Ther. 33, e13986 (2020).

8. Gianotti, R. SARS-CoV-2 and the skin, a hidden treasure. Dermatol. Rep. 12, (2020).

9. Yan, R. et al. Structural basis for the recognition of SARS-CoV-2 by full-length human ACE2. Science $367,1444-+(2020)$.

10. Xue, X. et al. High Expression of ACE2 on Keratinocytes Reveals Skin as a Potential Target for SARSCoV-2. J. Invest. Dermatol. 141, 206-209.e1 (2021).

11. Sun, Y. et al. Skin is a potential host of SARS-CoV-2: A clinical, single-cell transcriptome-profiling and histologic study. J. Am. Acad. Dermatol. 83, 1755-1757 (2020).

12. Choo, S. W. et al. Are pangolins scapegoats of the COVID-19 outbreak-CoV transmission and pathology evidence? Conserv. Lett. 13, e12754 (2020).

13. Barh, D. et al. Multi-omics-based identification of SARS-CoV-2 infection biology and candidate drugs against COVID-19. Comput. Biol. Med. 126, 104051 (2020).

14. Choo, S. W. et al. Pangolin genomes and the evolution of mammalian scales and immunity. Genome Res. 26, 1312-1322 (2016).

15. Fischer, H., Tschachler, E. \& Eckhart, L. Pangolins Lack IFIH1/MDA5, a Cytoplasmic RNA Sensor That Initiates Innate Immune Defense Upon Coronavirus Infection. Front. Immunol. 11, 939 (2020). 
16. Trapnell, C. et al. Differential gene and transcript expression analysis of RNA-seq experiments with TopHat and Cufflinks. Nat. Protoc. 7, 562-578 (2012).

17. Blanco-Melo, D. et al. Imbalanced host response to SARS-CoV-2 drives development of COVID-19. Cell 181, 1036-1045.e9 (2020).

18. Yu, G., Wang, L.-G., Han, Y. \& He, Q.-Y. clusterProfiler: an R package for comparing biological themes among gene clusters. Omics J. Integr. Biol. 16, 284-287 (2012).

19. Altschul, S. F., Gish, W., Miller, W., Myers, E. W. \& Lipman, D. J. Basic local alignment search tool. J. Mol. Biol. 215, 403-410 (1990).

20. Kumar, S., Stecher, G., Li, M., Knyaz, C. \& Tamura, K. MEGA X: Molecular Evolutionary Genetics Analysis across Computing Platforms. Mol. Biol. Evol. 35, 1547-1549 (2018).

21. Edgar, R. C. MUSCLE: a multiple sequence alignment method with reduced time and space complexity. BMC Bioinformatics 5, 113 (2004).

22. Nomburg, J., Meyerson, M. \& DeCaprio, J. A. Pervasive generation of non-canonical subgenomic RNAs by SARS-CoV-2. Genome Med. 12, 108 (2020).

23. Kim, D. et al. The Architecture of SARS-CoV-2 Transcriptome. Cell 181, 914-921.e10 (2020).

24. Liu, P. et al. Are pangolins the intermediate host of the 2019 novel coronavirus (SARS-CoV-2)? PLoS Pathog. 16, e1008421 (2020).

25. Masters, P. S. The molecular biology of coronaviruses. in Advances in Virus Research, Vol 66 (eds. Maramorosch, K. \& Shatkin, A. J.) vol. 66 193-+ (Elsevier Academic Press Inc, 2006).

26. Nakagawa, K., Lokugamage, K. G. \& Makino, S. Viral and Cellular mRNA Translation in CoronavirusInfected Cells. in Advances in Virus Research, Vol 96: Coronaviruses (ed. Ziebuhr, J.) vol. 96 165-192 (Elsevier Academic Press Inc, 2016).

27. Thoms, M. et al. Structural basis for translational shutdown and immune evasion by the Nsp1 protein of SARS-CoV-2. Science 369, 1249-+ (2020).

28. Schubert, K. et al. SARS-CoV-2 Nsp1 binds the ribosomal mRNA channel to inhibit translation. Nat. Struct. Mol. Biol. doi:10/ghrkth.

29. Li, S. Regulation of Ribosomal Proteins on Viral Infection. Cells 8, (2019).

30. Peltz, S. W. et al. Ribosomal protein L3 mutants alter translational fidelity and promote rapid loss of the yeast killer virus. Mol. Cell. Biol. 19, 384-391 (1999).

31. Leh, V., Yot, P. \& Keller, M. The cauliflower mosaic virus translational transactivator interacts with the 60S ribosomal subunit protein L18 of Arabidopsis thaliana. Virology 266, 1-7 (2000).

32. Spurgers, K. B. et al. Identification of essential filovirion-associated host factors by serial proteomic analysis and RNAi screen. Mol. Cell. Proteomics MCP 9, 2690-2703 (2010).

33. Li, S., Li, X. \& Zhou, Y. Ribosomal protein $L 18$ is an essential factor that promote rice stripe virus accumulation in small brown planthopper. Virus Res. 247, 15-20 (2018).

34. Cervantes-Salazar, M. et al. Dengue virus NS1 protein interacts with the ribosomal protein RPL18: this interaction is required for viral translation and replication in Huh-7 cells. Virology 484, 113-126 
(2015).

35. Martínez, F. \& Daròs, J.-A. Tobacco etch virus protein P1 traffics to the nucleolus and associates with the host 60S ribosomal subunits during infection. J. Virol. 88, 10725-10737 (2014).

36. Beyer, A. R. et al. Nucleolar trafficking of the mouse mammary tumor virus gag protein induced by interaction with ribosomal protein L9. J. Virol. 87, 1069-1082 (2013).

37. Leopardi, R. \& Roizman, B. Functional interaction and colocalization of the herpes simplex virus 1 major regulatory protein ICP4 with EAP, a nucleolar-ribosomal protein. Proc. Natl. Acad. Sci. U. S. A. 93, 4572-4576 (1996).

38. Jassal, B. et al. The reactome pathway knowledgebase. Nucleic Acids Res. 48, D498-D503 (2020).

39. Subramanian, A. et al. Gene set enrichment analysis: a knowledge-based approach for interpreting genome-wide expression profiles. Proc. Natl. Acad. Sci. U. S. A. 102, 15545-15550 (2005).

40. Day, S. L., Ramshaw, I. A., Ramsay, A. J. \& Ranasinghe, C. Differential effects of the type I interferons alpha4, beta, and epsilon on antiviral activity and vaccine efficacy. J. Immunol. Baltim. Md 1950 180, 7158-7166 (2008).

41. Pontén, F., Jirström, K. \& Uhlen, M. The Human Protein Atlas--a tool for pathology. J. Pathol. 216, 387-393 (2008).

42. Xi, Y., Day, S. L., Jackson, R. J. \& Ranasinghe, C. Role of novel type I interferon epsilon in viral infection and mucosal immunity. Mucosal Immunol. 5, 610-622 (2012).

43. Fung, K. Y. et al. Interferon- $\varepsilon$ protects the female reproductive tract from viral and bacterial infection. Science 339, 1088-1092 (2013).

44. Demers, A. et al. The mucosal expression pattern of interferon- $\varepsilon$ in rhesus macaques. J. Leukoc. Biol. 96, 1101-1107 (2014).

45. Uhlén, M. et al. Proteomics. Tissue-based map of the human proteome. Science 347, 1260419 (2015).

46. Guo, Y. et al. Molecular and functional characterization of ovis aries IFN-epsilon. Mol. Immunol. 119, 1-7 (2020).

47. Gendrot, M. et al. Antimalarial drugs inhibit the replication of SARS-CoV-2: An in vitro evaluation. Travel Med. Infect. Dis. 37, 101873 (2020).

48. Yan, B. et al. Characterization of the Lipidomic Profile of Human Coronavirus-Infected Cells: Implications for Lipid Metabolism Remodeling upon Coronavirus Replication. Viruses 11, (2019).

49. Kadkhoda, K. COVID-19: an Immunopathological View. mSphere 5, (2020).

50. Haljasmägi, L. et al. Longitudinal proteomic profiling reveals increased early inflammation and sustained apoptosis proteins in severe COVID-19. Sci. Rep. 10, 20533 (2020).

51. Henderson, L. A. et al. On the Alert for Cytokine Storm: Immunopathology in COVID-19. Arthritis Rheumatol. Hoboken NJ 72, 1059-1063 (2020).

52. Bagchi, P. Endoplasmic reticulum in viral infection. Int. Rev. Cell Mol. Biol. 350, 265-284 (2020). 
53. Chauhan, A. J., Wiffen, L. J. \& Brown, T. P. COVID-19: A collision of complement, coagulation and inflammatory pathways. J. Thromb. Haemost. JTH 18, 2110-2117 (2020).

54. Su, M. et al. A Mini-Review on Cell Cycle Regulation of Coronavirus Infection. Front. Vet. Sci. 7, 586826 (2020).

55. Yuan, X. et al. SARS coronavirus 7a protein blocks cell cycle progression at G0/G1 phase via the cyclin D3/pRb pathway. Virology 346, 74-85 (2006).

56. Yuan, X. et al. G1 phase cell cycle arrest induced by SARS-CoV 3a protein via the cyclin D3/pRb pathway. Am. J. Respir. Cell Mol. Biol. 37, 9-19 (2007).

57. Yuan, L. et al. p53 degradation by a coronavirus papain-like protease suppresses type I interferon signaling. J. Biol. Chem. 290, 3172-3182 (2015).

58. Ma-Lauer, Y. et al. p53 down-regulates SARS coronavirus replication and is targeted by the SARSunique domain and PLpro via E3 ubiquitin ligase RCHY1. Proc. Natl. Acad. Sci. U. S. A. 113, E51925201 (2016).

59. Wurm, T. et al. Localization to the nucleolus is a common feature of coronavirus nucleoproteins, and the protein may disrupt host cell division. J. Virol. 75, 9345-9356 (2001).

60. Chen, H., Wurm, T., Britton, P., Brooks, G. \& Hiscox, J. A. Interaction of the coronavirus nucleoprotein with nucleolar antigens and the host cell. J. Virol. 76, 5233-5250 (2002).

61. Cawood, R., Harrison, S. M., Dove, B. K., Reed, M. L. \& Hiscox, J. A. Cell cycle dependent nucleolar localization of the coronavirus nucleocapsid protein. Cell Cycle Georget. Tex 6, 863-867 (2007).

62. S, L. et al. Spring viraemia of carp virus modulates p53 expression using two distinct mechanisms. PLoS pathogens vol. 15 https://pubmed.ncbi.nlm.nih.gov/30925159/ (2019).

63. S, L. et al. The Severe Fever with Thrombocytopenia Syndrome Virus NSs Protein Interacts with CDK1 To Induce G 2 Cell Cycle Arrest and Positively Regulate Viral Replication. Journal of virology vol. 94 https://pubmed.ncbi.nlm.nih.gov/31852787/ (2020).

64. Belyi, V. A., Levine, A. J. \& Skalka, A. M. Unexpected Inheritance: Multiple Integrations of Ancient Bornavirus and Ebolavirus/Marburgvirus Sequences in Vertebrate Genomes. Plos Pathog. 6, e1001030 (2010).

65. Pinheiro, A. et al. An overview of the lagomorph immune system and its genetic diversity. Immunogenetics 68, 83-107 (2016).

66. Katzourakis, A. \& Aswad, A. Evolution: Endogenous Viruses Provide Shortcuts in Antiviral Immunity. Curr. Biol. CB 26, R427-429 (2016).

67. Grandi, N. \& Tramontano, E. Human Endogenous Retroviruses Are Ancient Acquired Elements Still Shaping Innate Immune Responses. Front. Immunol. 9, 2039 (2018).

68. Ziegler, C. G. K. et al. SARS-CoV-2 Receptor ACE2 Is an Interferon-Stimulated Gene in Human Airway Epithelial Cells and Is Detected in Specific Cell Subsets across Tissues. Cell 181, 1016-+ (2020).

69. Qi, F., Qian, S., Zhang, S. \& Zhang, Z. Single cell RNA sequencing of 13 human tissues identify cell types and receptors of human coronaviruses. Biochem. Biophys. Res. Commun. 526, 135-140 
(2020).

70. Li, Y. et al. The MERS-CoV Receptor DPP4 as a Candidate Binding Target of the SARS-CoV-2 Spike. iScience 23, 101160 (2020).

71. Solerte, S. B., Di Sabatino, A., Galli, M. \& Fiorina, P. Dipeptidyl peptidase-4 (DPP4) inhibition in COVID19. Acta Diabetol. 57, 779-783 (2020).

72. Gardinassi, L. G., Souza, C. O. S., Sales-Campos, H. \& Fonseca, S. G. Immune and Metabolic Signatures of COVID-19 Revealed by Transcriptomics Data Reuse. Front. Immunol. 11, (2020).

\section{Declarations}

\section{Acknowledgment}

The project is a part of the International Pangolin Research Consortium. We would like to thank the Guangzhou Leader Animal Hospital, the State Forestry and Grassland administration and Guangdong Provincial Forestry Administration for support throughout this study. This work was funded by the highlevel talent recruitment programme for academic and research platform construction (Reference Number: 5000105) from Wenzhou-Kean University.

\section{Author contributions}

S.W.C. conceived this project. S.W.C., S.Z., X.T, and J.Z performed animal sampling. S.W.C., X.T. designed and performed RNA extraction, library preparation, and sequencing experiments. S.D., S.W.C, H.C., W.C., H.Q. performed data analyses. S.W.C, S.D. performed data interpretation. S.D., S.W.C., R.B., Y.Y., X.T. wrote and revised the manuscript. All authors proofread and approved the manuscript.

\section{Competing interests}

No conflict of interest declared.

\section{Data accessibility}

The sequencing data has been deposited in the CNSA (https://db.cngb.org/cnsa/) of CNGBdb with accession number CNP0001573. Supporting data is included in the published paper and/or the additional information. For the additional information, it can be made to the corresponding author at cwoh@wku.edu.cn. 
"Animal ethics approval: This work was approved (reference number: GF(2019)BASE08) by the Biology and Science Ethics Committee of the China Biodiversity Conservation and Green Development Foundation (CBCGDF)."

\section{Figures}

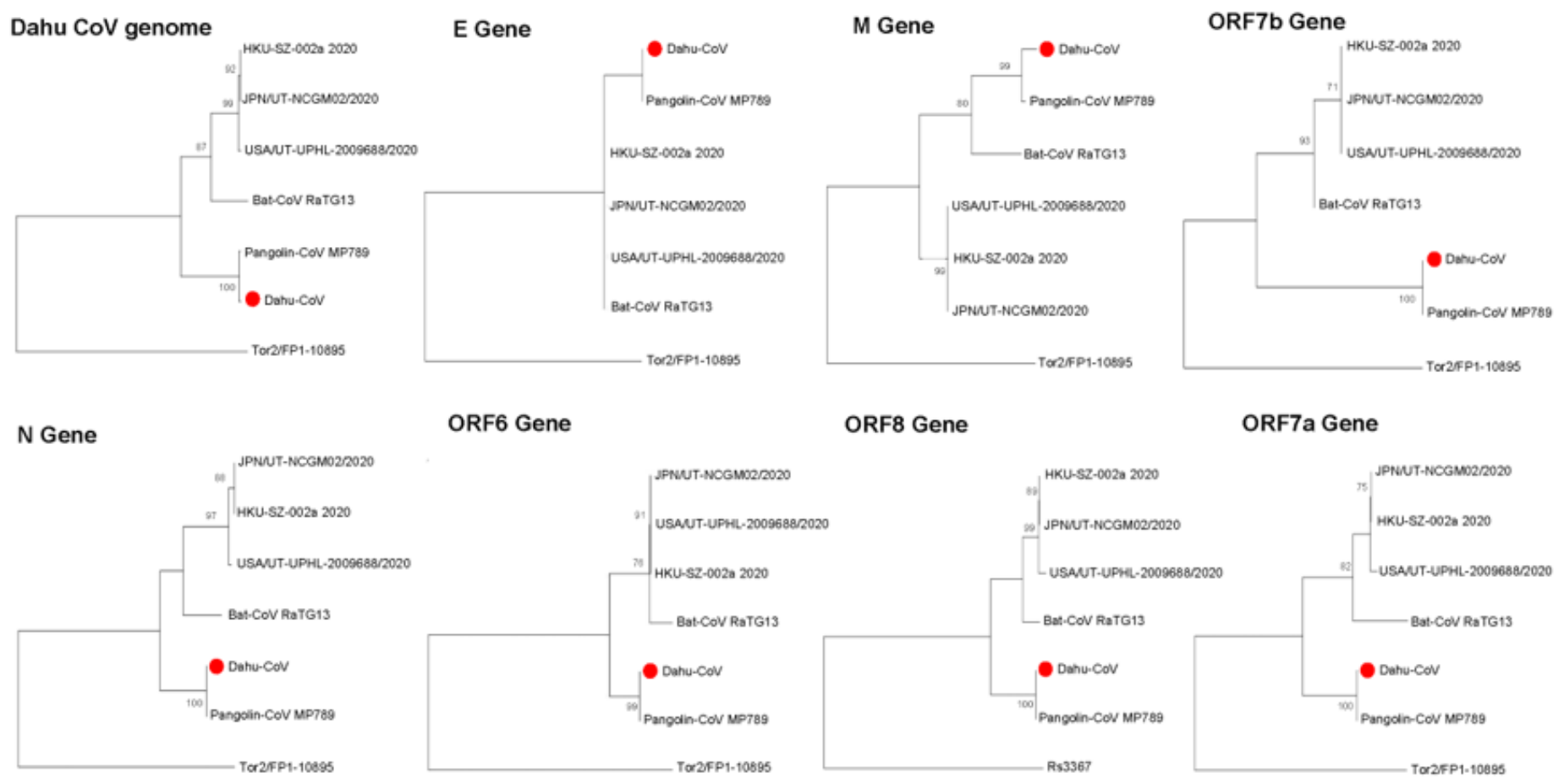

\section{Figure 1}

Phylogenetic trees of the coronavirus RNA and gene sequences from Dahu skin. The phylogenetic tree was generated using the maximum likelihood method. Bootstrap numbers were generated in 1,000 replicates. Nodes with bootstrap support values of 70 or greater are indicated. 
A

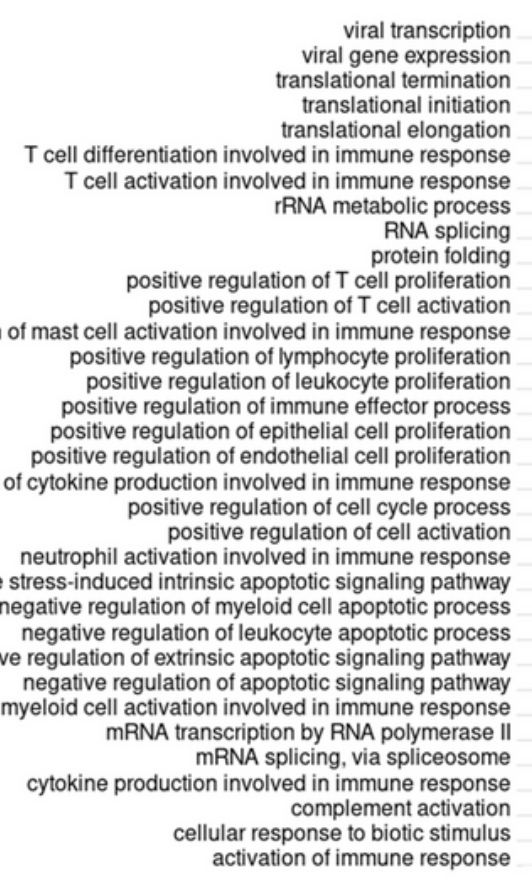
negative regulation of oxidative stress-induced intrinsic apoptotic signaling pathway negative regulation of myeloid cell apoptotic process
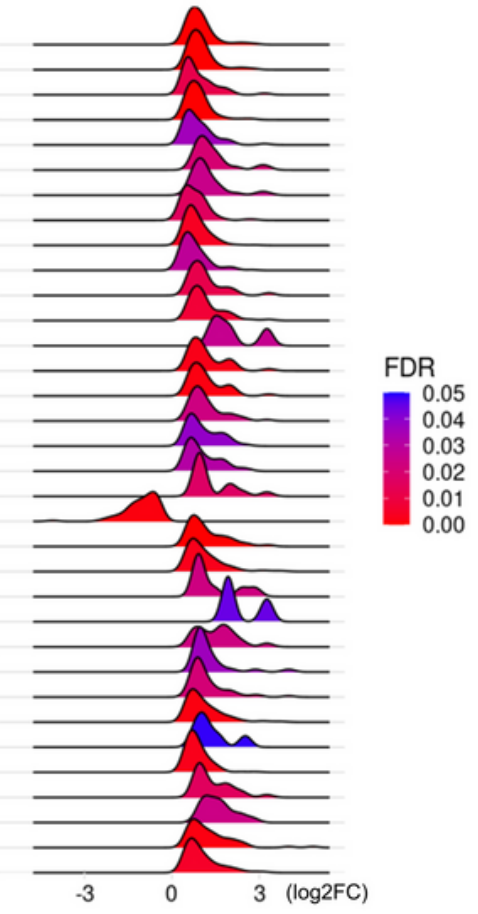

B

Wnt signaling pathway Vitamin digestion and absorption Viral protein interaction with cytokine and cytokine receptor Tuberculosis TNF signaling pathway

Steroid biosynthesis Sphingolipid metabolism Ribosome

Rheumatoid arthritis PI3K-Akt signaling pathway

Pertussis

Pathways in cancer

Pancreatic secretion Osteoclast differentiation

Nicotine addiction

Mineral absorption MAPK signaling pathway Malaria

Linoleic acid metabolism Leukocyte transendothelial migration Leishmaniasis

Human papillomavirus infection Glycerophospholipid metabolism
Focal adhesion

Fluid shear stress and atherosclerosis Epstein-Barr virus infection ECM-receptor interaction Cytokine-cytokine receptor interaction Coronavirus disease - COVID-19 Complement and coagulation cascades

Chemokine signaling pathway Cell adhesion molecules Bile secretion

alpha-Linolenic acid metabolism AGE-RAGE signaling pathway in diabetic complications

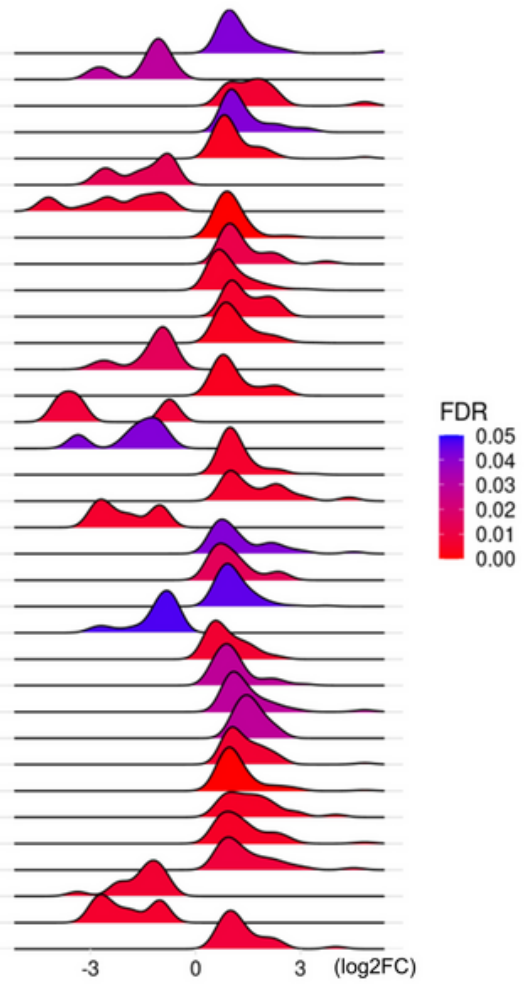

Figure 2

GSEA of pangolin genes. Ridge plots of (A) key GO BP terms and (B) KEGG pathways. GO, gene ontology; $\mathrm{BP}$, biological process; GSEA, gene set enrichment analysis; FDR, false discovery rate adjusted p-value. 
A

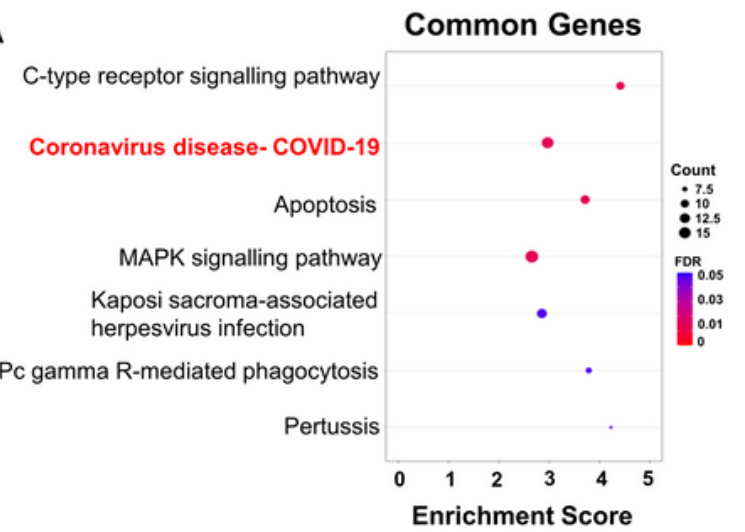

B

\section{Dahu Skin-specific Genes}

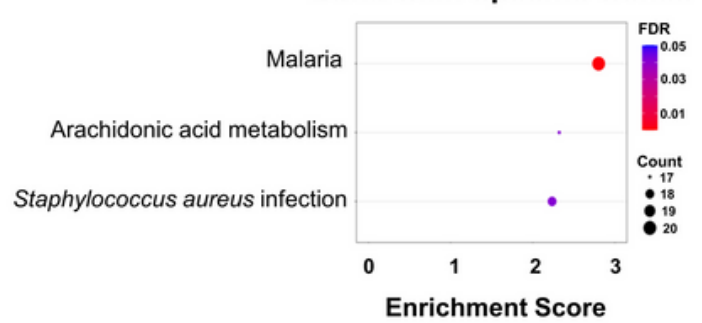

c

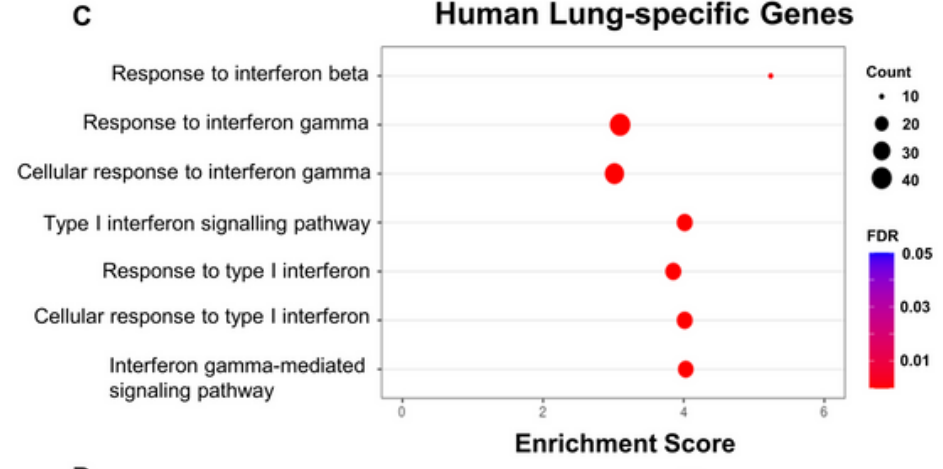

D

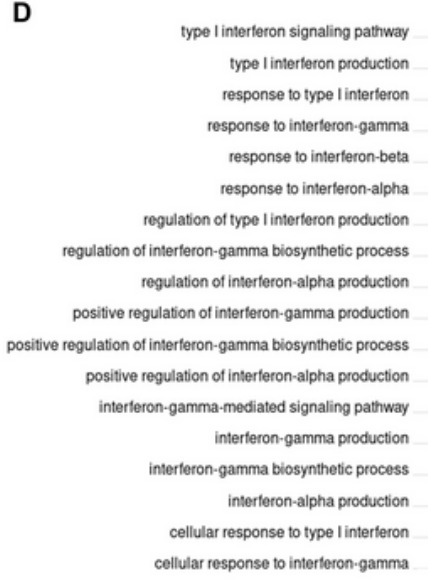

E

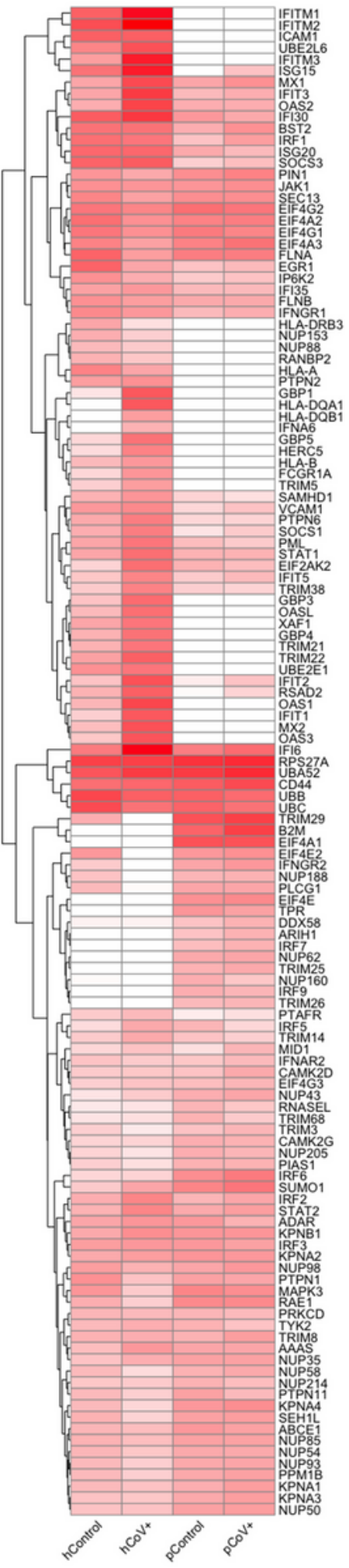

\section{Figure 3}

Comparative analysis and interferon-related responses were specifically up-regulated in human lungs, but not in pangolin skin with pCoV infection. (A) Enriched pathways in common DEGs between Dahu skin and human lung. (B) Enriched pathways in Dahu skin-specific DEGs. (C) Interferon-specific responses significantly enriched in human-specific DEGs. (D) Significant interferon-related terms from the human GSEA results. (E) Interferon pathway related gene expressions in healthy human lungs (hControl), SARS- 
CoV-2 infected human lungs ( $\mathrm{hCoV}+$ ), healthy pangolin skin (pControl), and pCoV infected pangolin skin $(\mathrm{pCoV}+)$. Gene expression in FPKM were log10 transformed and only expressed genes are shown. GO, gene ontology; BP, biological process; DEGs, differentially expressed genes; GSEA, gene set enrichment analysis; FDR, false discovery rate adjusted p-value; KEGG, Kyoto Encyclopedia of Genes and Genomes.

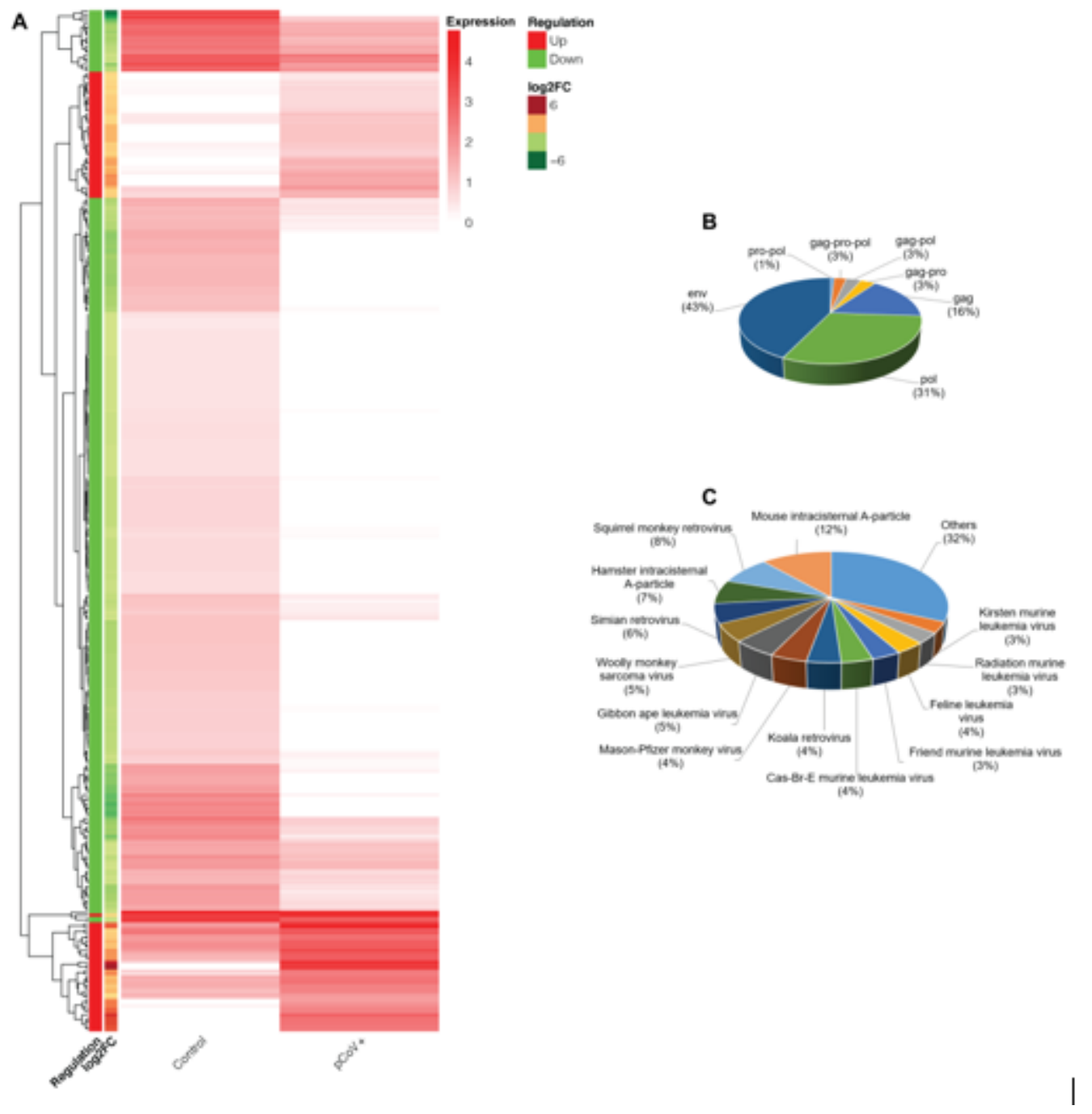

Figure 4

Differential expressed (DE) ERV gene composition and expression profiles. (A) ERV DEG expressions. (B) Breakdown of ERV DEG types. (C) Breakdown of retroviral types of ERV DEGs. The gene expressions in FPKM were log2 transformed before being divided by two. Retroviral names are taken from the closest match in our library of viral gene sequences.

\section{Supplementary Files}

This is a list of supplementary files associated with this preprint. Click to download.

- Supplementarylnformation.docx

- TableS1.xIsx 
- Tables2.xlsx

- Tables3.xIsx

- Tables4.xlsx

- Tables5.xIsx

- TableS6.xlsx

- TableS7.xlsx

- Tables8.xIsx 